\title{
Tunneling time through a barrier using the local value of a "time" operator
}

\author{
Donald H. Kobe, ${ }^{*}$ Hiromi Iwamoto, ${ }^{\dagger}$ Mario Goto ${ }^{\dagger}$ and Valdir C. Aguilera-Navarro \\ Department of Physics, University of North Texas, Denton, Texas 76203-1427 \\ (Received 10 February 2000; revised manuscript received 24 July 2000; published 17 July 2001)
}

\begin{abstract}
A time for a quantum particle to traverse a barrier is obtained for stationary states by setting the local value of a "time" operator equal to a constant. This time operator, called the tempus operator because it is distinct from the time of evolution, is defined as the operator canonically conjugate to the energy operator. The local value of the tempus operator gives a complex time for a particle to traverse a barrier. The method is applied to a particle with a semiclassical wave function, which gives, in the classical limit, the correct classical traversal time. It is also applied to a quantum particle tunneling through a rectangular barrier. The resulting complex tunneling time is compared with complex tunneling times from other methods.
\end{abstract}

DOI: 10.1103/PhysRevA.64.022104

PACS number(s): 03.65.Ta, 03.65.Sq

\section{INTRODUCTION}

Quantum mechanics has answered many questions about the microscopic world, but some questions still remain. One such question, addressed in this paper, is how long does it take for a quantum particle to tunnel through a classical forbidden region? Two related questions are (1) how long does a quantum particle spend in a classically forbidden region, and (2) how long does it take for a quantum particle to be reflected from a classically forbidden region? Although there has been intense interest in answering these questions because of the need to design nanoscale electronic devices using semiconductor heterostructures, no consensus has yet emerged [1-7].

In classical mechanics, a traversal time between two points in one dimension can easily be calculated. The trajectory of a particle in one dimension, $x=x(t)$, is calculated from Newton's second law and initial conditions. Assuming that we can solve the trajectory for the time $t=t(x)$, which we call the "inverse trajectory," we find that the traversal time $\Delta t=t\left(x_{2}\right)-t\left(x_{1}\right)$ is the time it takes for a classical particle to travel from $x_{1}$ to $x_{2}$.

In the usual interpretation of quantum mechanics, there is no particle trajectory, so the classical procedure cannot be applied directly. However, the de Broglie-Bohm causal interpretation of quantum mechanics [8] does give trajectories, and this point of view has been used by Leavens and coworkers [9-14] to address the tunneling time problem. The Feynman path-integral formulation of quantum mechanics has also been used to obtain tunneling times [15-18].

In this paper we propose another procedure for relating $x$ to $t$ in quantum mechanics and hence obtaining traversal or

\footnotetext{
*Email address: kobe@unt.edu

†Permanent address: Departamento de Física/CCE, Universidade Estadual do Londrina, 86051-990 Londrina, PR, Brazil. Email address: hiromi@uel.br,mgoto@uel.br

*Permanent address: Departamento de Química e Física, Universidade Estadual do Centro Oeste, 85015-430 Guarapuava, PR, Brazil, and Instituto de Física Teórica, Universidade Estadual Paulista, 01405-900 São Paulo, SP, Brazil. Email address: aguilera@ift.unesp.br
}

tunneling times. The procedure uses a quantum-mechanical operator, called the tempus operator $\hat{T}$ [19-21], that is canonically conjugate to the energy operator $\hat{E}$. Since tempus is different conceptually from the time $t$ of evolution of the system, we distinguish the two by using the Latin term for time, tempus. In the title, however, we have chosen to use "time" instead of tempus operator for the sake of clarity, since the tempus operator is not well known. Together the energy operator and the tempus operator satisfy the canonical commutation relation. The time $t$ of evolution in quantum mechanics remains a parameter.

We use the simplest local value of the tempus operator in quantum mechanics to obtain a traversal or a tunneling time. In the classical limit, the quantum traversal time reduces to its classical value. A local value of an operator is the operator acting on a wave function at a point in space and time, divided by the same wave function [22,23]. For the tempus operator the classical limit of this local value gives a constant, which is the classical value given by the HamiltonianJacobi theory $[8,24]$. Our procedure postulates that even in the quantum case, a local value of the tempus operator is a constant and formulates this as a "principle of constant local tempus." Using this principle, we obtain an "inverse quasitrajectory" $t(x)$ that is the inverted "quasitrajectory" $x(t)$ for a quantum-mechanical particle in one dimension. From the inverse quasitrajectory $t(x)$ for a particle we can calculate a traversal time $\tau=\Delta t=t\left(x_{2}\right)-t\left(x_{1}\right)$ between the points $x_{1}$ to $x_{2}$. A traversal time for a particle in the classically forbidden region of a barrier is called a tunneling time. Our method gives a complex tunneling time in which the real part is called a phase time and the imaginary part is called a transmission time. This complex tunneling time is similar to one obtained by Pollak and Miller [25] using a flux-flux correlation function. An experiment on frustrated total internal reflection of light used the complex Pollak-Miller time and interpreted its real and imaginary parts [26].

The tunneling time proposed here is only one contribution to a long list of proposals for calculating a tunneling time through a barrier [1-7]. In quantum mechanics there may be several versions of a quantity that in classical mechanics is unique, and these different versions may be realized in different experiments. A universal intrinsic tunneling time that is valid for all experiments probably does not exist. There are 
most likely a multiplicity of tunneling times in nature, each one describing a different type of experiment. Tunneling time experiments that use some type of clock may depend on the type of clock used $[16,27,28]$. The calculated tunneling time that applies to a given experimental setup is a question that can only be answered by more experiments $[29,30]$.

In this paper the tempus operator is discussed in Sec. II. In Sec. III the classical limit of the local value of the tempus operator is shown to be constant. Section IV generalizes this result to the quantum case and postulates a "principle of constant local tempus" for stationary state wave functions that gives a complex traversal time. We apply this principle in Sec. V to calculate a traversal time in a semiclassical (WKB) approximation for a particle with an energy large compared to the height of the barrier that agrees with the classical traversal time. In Sec. VI we apply this principle to a rectangular barrier to calculate a tunneling time and compare it with the Pollak-Miller time [25] and the Larmor time $[31,32]$. The conclusion is given in Sec. VII.

\section{TEMPUS OPERATOR}

The concept of tempus is defined in classical Hamiltonian mechanics $[19,20]$ as the variable, with dimensions of time, that is canonically conjugate to the energy. In classical Hamiltonian mechanics a canonical transformation can be made from old canonical variables $(q, p)$ to new canonical variables $\left(q^{\prime}, p^{\prime}\right)$, where $q\left(q^{\prime}\right)$ and $p\left(p^{\prime}\right)$ are the old (new) generalized coordinate and the canonical momentum conjugate to it, respectively. If we now choose the new canonical momentum $p^{\prime}$ to be the energy $E$, the generalized coordinate $q^{\prime}$ conjugate to it is called tempus, denoted by $T[19,20]$. Because energy and tempus are canonically conjugate variables, they satisfy the same Poisson bracket as $q$ and $p$ satisfy

$$
\{T, E\}=1 .
$$

Since the Poisson bracket is a canonical invariant [24], it can be calculated with respect to any set of canonically conjugate coordinates and momenta. In general, tempus $T=T(q, E, t)$ is a function of the old generalized coordinate $q$, the new canonical momentum $p^{\prime}=E$, and the time of evolution $t$. An arbitrary function of the energy can be added to tempus so it is not unique, but for simplicity we take this arbitrary function to be zero. In a conservative system tempus is numerically equal to the time of evolution $t$ plus a constant, but conceptually they are different. When the system is quantized we also quantize tempus [21], but not the time of evolution $t$ that parametrizes the wave function. The distinction between tempus $T$ and the time of evolution $t$ provides a basis for an operator $\hat{T}$ in quantum mechanics that is different from the usual "time operators" $\hat{t}$ that quantize the time of evolution. Pauli [33] has objected to a time operator and has given a "proof" that it does not exist. Recent work uncovers some implicit assumptions made by Pauli and shows that a self-adjoint time operator can exist [34]. General time operators and time of arrival operators have also been recently discussed [35]. In some cases a time operator can be constructed that is not self-adjoint [36-38].
To quantize a classical system, we assume Dirac [39] quantization in which classical variables are replaced by operators and Poisson brackets are replaced by commutator

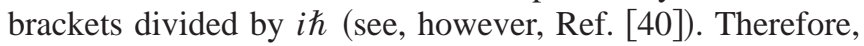
energy $E$ is replaced by an energy operator $\hat{E}$ and tempus $T$ is replaced by a tempus operator $\hat{T}$ on an appropriate domain. Together the two operators satisfy the standard canonical commutation relation of quantum mechanics [21]

$$
[\hat{T}, \hat{E}]=i \hbar,
$$

corresponding to the classical Poisson bracket in Eq. (1). An arbitrary function of the energy operator may be added to the tempus operator, but for simplicity it is taken to be zero. Various realizations of the energy and tempus operators that satisfy Eq. (2) are possible [21], but the realization used here is the one with the energy operator $\hat{E}=E$ and the corresponding tempus operator $\hat{T}=i \hbar \partial / \partial E$. Some of the other realizations of the energy and tempus operators in specific cases act directly on the coordinate of the wave function [21], and thus open the possibility of applying our method to a wave packet.

Razavy [41-43] has used a similar approach based on a "time function" in classical mechanics that is canonically conjugate to the Hamiltonian. He used it to calculate the time delay in scattering, but did not use it to calculate a tunneling time. Quantum canonical transformations have been used by León et al. [44].

The approach of Recami et al. [45-48] is to introduce a "time operator" $\hat{t}_{1}=-i \hbar \partial / \partial E$ and to calculate its expectation value with respect to a wave packet $f(x, E)$, where the integration is with respect to the energy $E>0$. This expectation value is equal to the expectation value of $t$ calculated with respect to the Fourier transformed wave packet $F(x, t)$, where the integration is with respect to time $t$ from $-\infty$ to $\infty$. Although this expectation value appears to be outside the usual Hilbert space interpretation of quantum mechanics in which time $t$ is considered a parameter and quantummechanical expectation values are calculated by integrating over all space, Egusquiza and Muga [35] have nevertheless shown that it can be written in standard quantum-mechanical form. Recami et al. [45-48] use their time operator to derive the energy-time uncertainty relation, time delay due to scattering, and decay from a metastable state.

The difficulty with using a time operator $\hat{t}$ is that time $t$ must also be a parameter in quantum mechanics to give the time evolution of the system. The use of the tempus operator $\hat{T}$ avoids this dual use of time, since it is conceptually different from the time of evolution $t$, which remains a parameter.

\section{CLASSICAL LIMIT OF LOCAL MOMENTUM AND} TEMPUS

We first examine a local value of the momentum operator and show that in the classical limit it is the classical momentum. We next consider a local value of the tempus operator and show that in the classical limit it is a constant. A local 
value of an operator is the operator acting on a wave function at a point in space and time divided by the same wave function $[22,23]$.

A wave function $\psi=\psi(x, t)$ can always be written in polar form

$$
\psi=\rho^{1 / 2} \exp (i S / \hbar)
$$

where $\rho$ is the probability density and $S / \hbar$ is the phase. When this expression is substituted into the time-dependent Schrödinger equation, the function $S(x, t)$ satisfies the Hamilton-Jacobi equation with a quantum potential added to the potential energy [8]. In the limit as $\hbar \rightarrow 0$, the quantum potential vanishes and the function $S$ becomes Hamilton's principal function $S_{\mathrm{HJ}}$.

\section{A. Local value of the momentum operator}

In order to gain more familiarity with local values, we first consider the local value of the momentum operator. Since the momentum operator in the coordinate representation is $\hat{p}=-i \hbar \partial / \partial x$, its local value at the spatial point $x$ at time $t$ is

$$
\frac{\hat{p} \psi}{\psi}=\frac{\partial S}{\partial x}-i \hbar \frac{1}{2} \frac{\partial \ln \rho}{\partial x},
$$

where Eq. (3) is used. The limit as $\hbar \rightarrow 0$ on the right-hand side of Eq. (4), becomes the classical momentum $p$

$$
\frac{\partial S_{\mathrm{HJ}}}{\partial x}=p
$$

from Hamilton-Jacobi theory [24].

For a plane wave with energy $E$ and momentum $p$, the wave function is $\psi=\psi_{0} \exp [i(-E t+p x) / \hbar]$, where $\psi_{0}$ is a constant amplitude. From Eq. (3), we obtain, in this case, $S$ $=-E t+p x$. The local value of the momentum in Eq. (4) gives $\partial S / \partial x=p$, the same as the classical momentum in Eq. (5).

\section{B. Local value of the tempus operator}

In a similar way, a local value of the tempus operator may be calculated when the wave function $\psi(x, t)$ describes a stationary state depending on energy $E$. When Eq. (3) is used for the wave function, the local value of the tempus operator $\hat{T}=i \hbar \partial / \partial E$ is

$$
\frac{\hat{T} \psi}{\psi}=-\frac{\partial S}{\partial E}+i \hbar \frac{1}{2} \frac{\partial \ln \rho}{\partial E} .
$$

In the limit as $\hbar \rightarrow 0$, the right-hand side of Eq. (6) becomes a constant, since

$$
\frac{\partial S}{\partial E} \rightarrow \frac{\partial S_{\mathrm{HJ}}}{\partial E}=\frac{\partial S_{\mathrm{HJ}}}{\partial p_{\mathrm{HJ}}}=q_{\mathrm{HJ}}, \text { a constant, }
$$

from Hamilton-Jacobi theory [24]. The derivative $\partial S_{\mathrm{HJ}} / \partial p_{\mathrm{HJ}}$, with respect to the new canonical momentum
$p_{\mathrm{HJ}}=E$ (a constant parameter) is the new conjugate coordinate $q_{\mathrm{HJ}}$ (another constant parameter).

For a plane wave $S=-E t+p x$ and $\psi_{0}$ is a constant. If we set the local value of the tempus in Eq. (6) equal to a constant in this case, we obtain

$$
-\frac{\partial S}{\partial E}=t-\frac{\partial p}{\partial E} x=\text { const. }
$$

Since $\partial E / \partial p=p / m=v$ is the velocity of the particle, Eq. (8) gives the classical trajectory for a free particle $x=x(t)=x_{0}$ $+v t$, where the constant is chosen such that $x_{0}$ is the initial position at $t=0$. The time $t_{21}=t_{2}-t_{1}$ for the free quantummechanical particle of energy $E$ to traverse the distance $x_{21}$ $=x_{2}-x_{1}$ from $x_{1}$ to $x_{2}$, is therefore $t_{21}=x_{21} / v$, the time it takes for a classical free particle of the same energy to traverse the same distance. This example shows that the postulate of a constant local tempus in Eq. (6) gives a meaningful result from which to calculate a traversal time.

\section{TRAVERSAL TIMES USING TEMPUS OPERATOR}

\section{A. Constant local value of tempus}

A natural generalization of the classical limit of Eq. (6) gives a procedure for relating $x$ to $t$ in quantum mechanics. We generalize the classical result in the simplest way and postulate that in quantum mechanics a local value of the tempus operator $\hat{T}=i \hbar \partial / \partial E$, calculated from a stationary state wave function $\psi(x, t)$, is still constant,

$$
\mathcal{T}(x, t) \equiv \frac{\hat{T} \psi(x, t)}{\psi(x, t)}=\mathrm{const}(\text { stationary state }),
$$

where the energy operator $E$ and tempus operator $\hat{T}$ $=i \hbar \partial / \partial E$ satisfy the commutation relation in Eq. (2) and the constant is, in general, complex. We call Eq. (9) the "principle of constant local tempus." It is a new principle for obtaining a quasitrajectory $x=x(t)$ in quantum mechanics and consequently an inverse quasitrajectory $t=t(x)$ from which a traversal time can be calculated. There are many possible local values that have the correct classical limit. We use the simplest one involving the tempus operator $\hat{T}$, even though the result is a complex constant. If a symmetrized form of the tempus operator were used, a real constant would be obtained [23]. Equation (9) is independent of the normalization of the wave function and is even valid for wave functions that are not normalizable, as for barrier problems. The local value of tempus $\mathcal{T}(x, t)$ is also invariant under a gauge transformation on the wave function $\psi^{\prime}(x, t)$ $=\exp [i \Lambda(x, t)] \psi(x, t)$, since an arbitrary gauge function $\Lambda(x, t)$ is independent of the energy.

The principle of constant local tempus is applicable to stationary-state solutions of the Schrödinger equation. In analogy with the classical case, we can calculate a tunneling time through a barrier from an "inverse quasitrajectory" $t$ $=t(x)$. For a particle with a potential energy $V(x)$, the timedependent Schrödinger equation has stationary-state solutions 


$$
\psi(x, t)=\psi(x) \exp (-i E t / \hbar),
$$

where the energy eigenvalue is $E$ and the energy eigenfunction is $\psi(x)=\psi_{E}(x)$. We shall consider a particle with positive energies in the continuum and eigenfunctions that are not normalizable.

When the stationary-state solution in Eq. (10) is substituted into Eq. (9), we obtain an explicit expression for the inverse quasitrajectory, $\mathcal{T}(x, t) \equiv t+\mathcal{T}(x)=$ const, or

$$
t(x)=-\frac{\hat{T} \psi(x)}{\psi(x)}+\text { const (stationary state). }
$$

Therefore, the time for the particle to traverse from a point $x_{1}$ to a point $x_{2}$ is

$$
\tau \equiv t\left(x_{2}\right)-t\left(x_{1}\right)=\Delta t=-\Delta \frac{\hat{T} \psi}{\psi},
$$

where $\Delta$ denotes the difference in values at $x_{2}$ and $x_{1}$. The time $\tau$ is independent of the constant in Eq. (11) and is also independent of any arbitrary energy-dependent function multiplying the wave function. If the particle has an energy less than the top of a barrier, the traversal time $\tau$ is a tunneling time, where the point $x_{1}$ is the entrance to the barrier and $x_{2}$ is the exit.

\section{B. Traversal time}

Since $\hat{T}=i \hbar \partial / \partial E$, the traversal time $\tau$ in Eq. (12) is

$$
\tau=-i \hbar \Delta \frac{\partial \ln \psi}{\partial E}
$$

which is in general complex. We can write the (energydependent) stationary-state wave function in terms of its modulus and phase

$$
\psi(x)=|\psi(x)| \exp [i \phi(x)],
$$

and substitute it into Eq. (13). The real part of $\tau$ is a phase time [49-51]

$$
\tau_{\text {phase }}=\hbar \frac{\partial \Delta \phi}{\partial E},
$$

where $\Delta \phi=\phi\left(x_{2}\right)-\phi\left(x_{1}\right)$ is the difference in the phase of the total wave function at $x_{2}$ and at $x_{1}$. The imaginary part of $\tau$ is called [25] a transmission time,

$$
\tau_{\text {trans }}=-\hbar \frac{\partial}{\partial E} \ln \left|\frac{\psi\left(x_{2}\right)}{\psi\left(x_{1}\right)}\right|,
$$

where the absolute value is the ratio of the total wave function at $x_{2}$ to its value at $x_{1}$.

For tunneling, Eq. (13) is similar to a complex time obtained by Pollak and Miller [25] on the basis of a flux-flux correlation function. The Larmor time obtained by Büttiker and Landauer [31] can also be written in a similar complex form [32]. Complex tunneling times have been obtained from the Feynman path-integral point of view [15-18]. Brouard, et al. [28] discuss different definitions of tunneling times and their interpretation.

\section{TRAVERSAL TIME IN A SEMICLASSICAL APPROXIMATION}

As an example of the method in Sec. IV, we use a semiclassical wave function to obtain the local momentum and the local tempus. A traversal time is calculated for a quantum particle with an energy greater than the barrier.

\section{A. Local momentum}

The WKB approximation gives a wave function that is valid in a semiclassical approximation. For a particle with an energy above the barrier, a (unnormalized) WKB wave function is

$$
\psi=k(x)^{-1 / 2} \exp \left\{i\left[\int^{x} d x^{\prime} \hbar k\left(x^{\prime}\right)-E t\right] / \hbar\right\},
$$

where the local wave number at the point $x$ is

$$
k(x)=\{2 m[E-V(x)]\}^{1 / 2} / \hbar .
$$

The wave function in Eq. (17) describes propagation, without reflection, to larger values of $x$ as time increases and is valid for energies large compared to the height of the barrier.

Using this wave function, we find that the local value of the momentum operator, $\hat{p}=-i \hbar \partial / \partial x$, is

$$
\frac{\hat{p} \psi}{\psi}=p(x)-\frac{i \hbar}{4} \frac{V^{\prime}(x)}{[E-V(x)]},
$$

where the local momentum is $p(x)=\hbar k(x)$. In the classical limit $\hbar \rightarrow 0$ (or in the limit of large $E$ ) only the real part $p(x)$ of Eq. (19) contributes, which is the classical momentum as a function of the position $x$.

\section{B. Local tempus}

The local value of the tempus operator is

$$
\frac{\hat{T} \psi}{\psi}=i \hbar \frac{\partial}{\partial E} \ln \psi=t-\int^{x} d x^{\prime} \frac{1}{v\left(x^{\prime}\right)}-\frac{i \hbar}{4} \frac{1}{E-V(x)}=\text { const. }
$$

In the classical limit $\hbar \rightarrow 0$, the imaginary part goes to zero. The local velocity $v(x)$ in Eq. (20) is

$$
v(x)=\left[\frac{\partial p(x)}{\partial E}\right]^{-1}=\frac{p(x)}{m},
$$

where the classical local momentum $p(x)=\hbar k(x)$ is defined by Eq. (18). This local velocity $v(x)$ is the same as the "hydrodynamic" velocity defined as the ratio of the probability current density $j(x)$ to the probability density $\rho(x)$

$$
v(x)=\frac{j(x)}{\rho(x)}=\frac{1}{\psi^{*} \psi} \operatorname{Re} \psi^{*} \frac{\hat{p}}{m} \psi=\frac{p(x)}{m} .
$$


Using the principle of constant local tempus in Eq. (20), we obtain the relation

$$
t(x)=\int^{x} d x^{\prime} \frac{1}{v\left(x^{\prime}\right)}+\frac{i \hbar}{4} \frac{1}{E-V(x)}+\text { const } .
$$

The real part of Eq. (23) gives the phase time in Eq. (15) for a quantum particle with an energy above the barrier to traverse from $x_{1}$ to $x_{2}$,

$$
\tau_{\text {phase } 21}=\int_{x_{1}}^{x_{2}} d x^{\prime} \frac{1}{v\left(x^{\prime}\right)},
$$

which is the traversal time for a classical particle. This result might have been expected, since we are using a semiclassical WKB wave function. The WKB approximation is valid if the energy of the particle is much greater than the height of the barrier. The transmission time for the traversal of the quantum particle with an energy much larger than the top of the barrier is of no significance.

\section{TUNNELING THROUGH A RECTANGULAR BARRIER}

As another example of the principle of constant local tempus, we calculate the tunneling time for a rectangular barrier. Then we compare it with other complex tunneling times.

\section{A. Solution of the Schrödinger equation}

The potential energy in the Schrödinger equation for a rectangular barrier of height $V_{0}$ and width $a$ is

$$
V(x)=\left\{\begin{array}{cc}
V_{0}, & \text { if } 0 \leqslant x \leqslant a \\
0, & \text { otherwise }
\end{array}\right.
$$

For this potential, the solution of the Schrödinger equation for the energy less than the barrier height $\left(E<V_{0}\right)$ is the wave function

$$
\psi(x)=\left\{\begin{array}{cc}
\exp (i k x)+A \exp (-i k x), & \text { for } x<0, \\
B \exp (\kappa x)+C \exp (-\kappa x), & \text { for } 0 \leqslant x \leqslant a, \\
D \exp (i k x), & \text { for } x>a .
\end{array}\right.
$$

The wave number $k$ for the incident and reflected wave functions $(x<0)$, and the transmitted wave function $(x>a)$ is

$$
k=(2 m E)^{1 / 2} / \hbar
$$

the imaginary wave number in the forbidden region $(0 \leqslant x$ $\leqslant a)$ is

$$
\kappa=\left[2 m\left(V_{0}-E\right)\right]^{1 / 2} / \hbar,
$$

and a characteristic wave number $k_{0}$ is defined as

$$
k_{0}=\left(2 m V_{0}\right)^{1 / 2} / \hbar
$$

such that $k^{2}+\kappa^{2}=k_{0}^{2}$. The wave function in Eq. (26) is not normalizable when integrated over the whole real axis, but this poses no difficulty in determining the tunneling time.
The choice of coefficient for the incident wave is irrelevant, since only the ratio of the other coefficients to the coefficient of the incident wave can be calculated from the boundary conditions. Without loss of generality, we therefore choose the coefficient of the incident wave $\exp (i k x)$ in Eq. (26) to be unity. For energies greater than the height of the barrier $(E$ $\left.>V_{0}\right), \kappa$ in Eq. (28) is pure imaginary $\kappa=i k^{\prime}$, where $k^{\prime}$ $=\left[2 m\left(E-V_{0}\right)\right]^{1 / 2} / \hbar$ is a real wave number.

The boundary conditions that the wave function and its derivative are continuous at points 0 and $a$, are used to obtain the coefficients in Eq. (26). The complex transmission amplitude $D$ is

$$
D=2 \kappa k N^{-1} \exp \{-i(\alpha+k a-\pi / 2)\},
$$

where the unit of length is $k_{0}^{-1}$, the angle $\alpha$ is

$$
\alpha=\arctan [\operatorname{coth}(\kappa a) \tan (2 \theta)],
$$

and the angle $\theta=\arctan (\kappa / k)$. The complex reflection amplitude $A$ is

$$
A=\sinh (\kappa a) N^{-1} \exp (-i \alpha)
$$

where the function $N$ in Eqs. (30) and (32) is

$$
N=\left[\sinh ^{2}(\kappa a)+(2 \kappa k)^{2}\right]^{1 / 2}
$$

Equations (30) and (32) are used to calculate the phase and transmission times.

\section{B. Phase time}

The phase time in Eq. (15) requires the difference in the phase between wave function in Eq. (26) at the exit $x_{2}=a$ and the entrance $x_{1}=0$, which is

$$
\begin{aligned}
\Delta \phi= & (-\alpha+\pi / 2)-\arctan \left(\frac{A_{i}}{1+A_{r}}\right) \\
= & -\arctan \left[\frac{2 \kappa k}{k^{2}-\kappa^{2}} \operatorname{coth}(\kappa a)\right]+\frac{\pi}{2} \\
& +\arctan \left[\frac{\kappa k \sinh (2 \kappa a)}{(2 \kappa k)^{2}+2 k^{2} \sinh ^{2}(\kappa a)}\right],
\end{aligned}
$$

where $A_{r}$ and $A_{i}$ are the real and imaginary parts of $A$ in Eq. (32), respectively. The phase time calculated from Eq. (15) is therefore

$$
\tau_{\text {phase }}=\frac{2 k^{2} \kappa a-\sinh (2 \kappa a)}{4 \kappa k\left[k^{2}-\cosh ^{2}(\kappa a)\right]} .
$$

In Eq. (35) and throughout the rest of this paper, the unit of length is $k_{0}^{-1}$, the unit of energy is $V_{0}$, and the unit of time is $\tau_{0}=\hbar / V_{0}$.

Equation (35) for $\tau_{\text {phase }}$ is plotted as the solid curve in Fig. 1 as a function of the energy $E$, and in Fig. 2 as a function of barrier width $a$. The peaks in Fig. 1 have no simple relationship with the corresponding wavelength. Figure 2 shows that the tunneling time saturates for values of the width $a k_{0}>5$, 


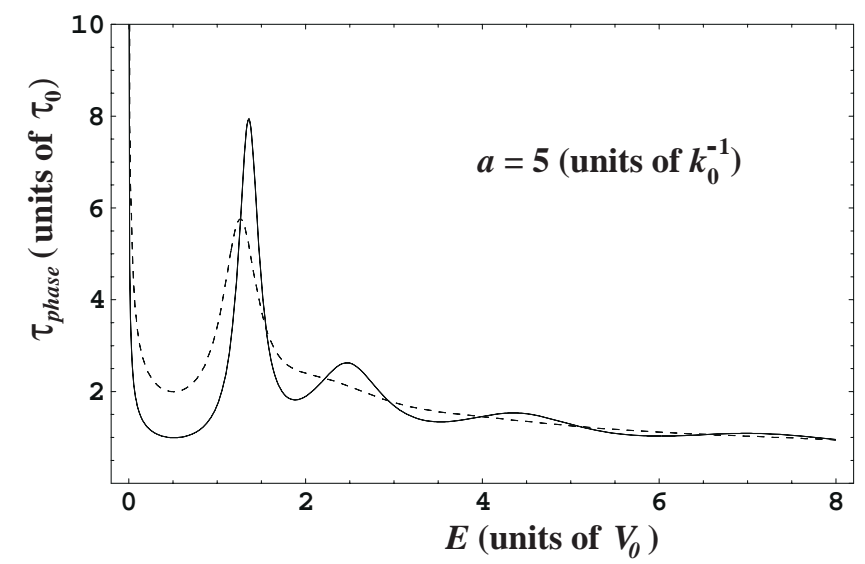

FIG. 1. Phase time $\tau_{\text {phase }}$ (units of $\tau_{0}$ ) for the rectangular barrier as a function of energy $E$ (units of $V_{0}$ ) for a barrier width $a=5$ (units of $k_{0}^{-1}$ ). The solid line is our result in Eq. (35) and the dashed line is the result of Pollak and Miller in Eq. (38).

called the "Hartman effect" [52]. From Eq. (35), the phase time saturates at a value $\tau_{\text {phase }}=(2 \kappa k)^{-1}$, as $a k_{0} \rightarrow \infty$. For the value of the energy $E=0.5$ given in Fig. 2, the saturation value is 1 . The phase time in Fig. 2 as a function of $a$ has an initial slope of $(2 k)^{-1}$, which for the value of the energy $E=0.5$ given in Fig. 2 , is $1 / \sqrt{2}$.

\section{Transmission time}

The imaginary part of $\tau$ in Eq. (13) is the transmission time $\tau_{\text {trans }}$ in Eq. (16). Substituting Eq. (26) into Eq. (16), we obtain

$$
\tau_{\text {trans }}=-\frac{1}{2} \frac{\partial}{\partial E} \ln \left|\frac{D}{1+A}\right|^{2}
$$

When Eqs. (30) and (32) are substituted into Eq. (36), the transmission time is

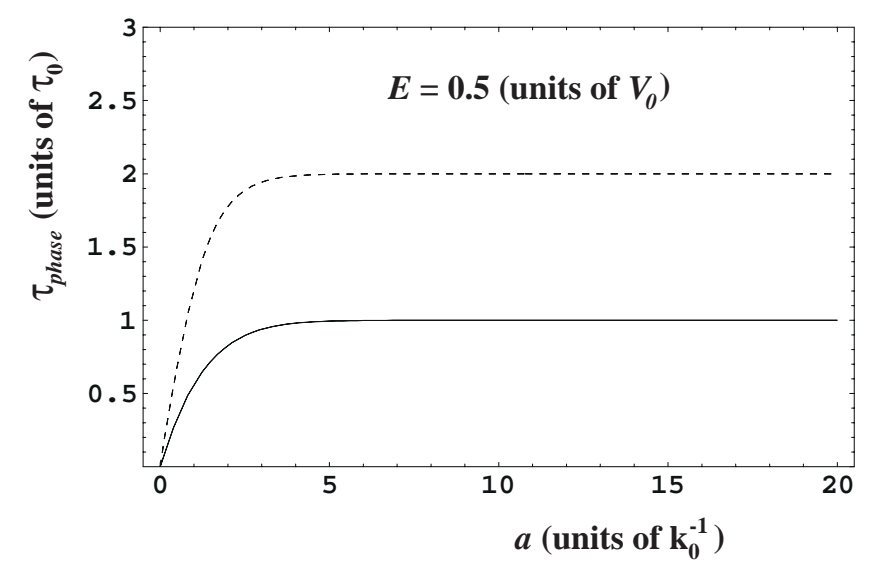

FIG. 2. Phase time $\tau_{\text {phase }}$ (units of $\tau_{0}$ ) for the rectangular barrier as a function of barrier width $a$ (units of $k_{0}^{-1}$ ) for an energy of $E$ $=0.5$ (units of $V_{0}$ ). The solid line is our result in Eq. (35) and the dashed line is the result of Pollak and Miller in Eq. (38).

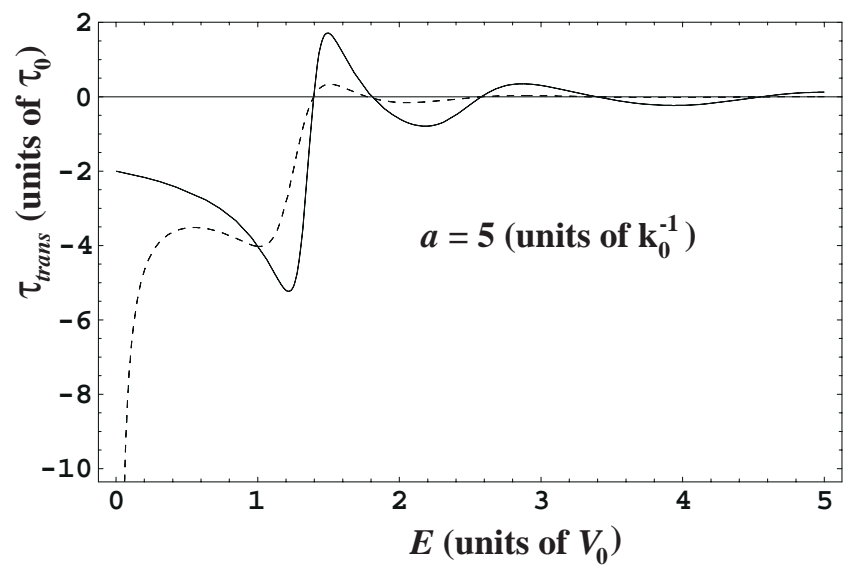

FIG. 3. Transmission time $\tau_{\text {phase }}$ (units of $\tau_{0}$ ) for the rectangular barrier as a function of energy $E$ (units of $V_{0}$ ) for a barrier width $a=5$ (units of $k_{0}^{-1}$ ). The solid line is our result in Eq. (37) and the dashed line is the result of Pollak and Miller in Eq. (39).

$$
\tau_{\text {trans }}=\frac{\sinh (\kappa a)[\kappa a \cosh (\kappa a)-\sinh (\kappa a)]}{2 \kappa^{2}\left[k^{2}-\cosh ^{2}(\kappa a)\right]} .
$$

Equation (37) for $\tau_{\text {trans }}$ is plotted as the solid curve in Fig. 3 as a function of the energy $E$, and in Fig. 4 as a function of the barrier width $a$. The negative values for $\tau_{\text {trans }}$ seem to be unphysical. Equation (37) shows that $\tau_{\text {trans }}$ is linear with a slope of $-(2 \kappa)^{-1}$ for widths $a k_{0} \gg 1$, which is shown in Fig. 4.

\section{Other tunneling times}

\section{Pollak-Miller time}

The Pollak-Miller time [25] is a complex tunneling time that was calculated from a flux-flux correlation function. The same tunneling time was obtained more simply by Leavens and Aers [32] by generalizing the method of the "energy sensitivity of the transmission amplitude" due to Büttiker and Landauer [53], and analyzed further by Martin and Lan-

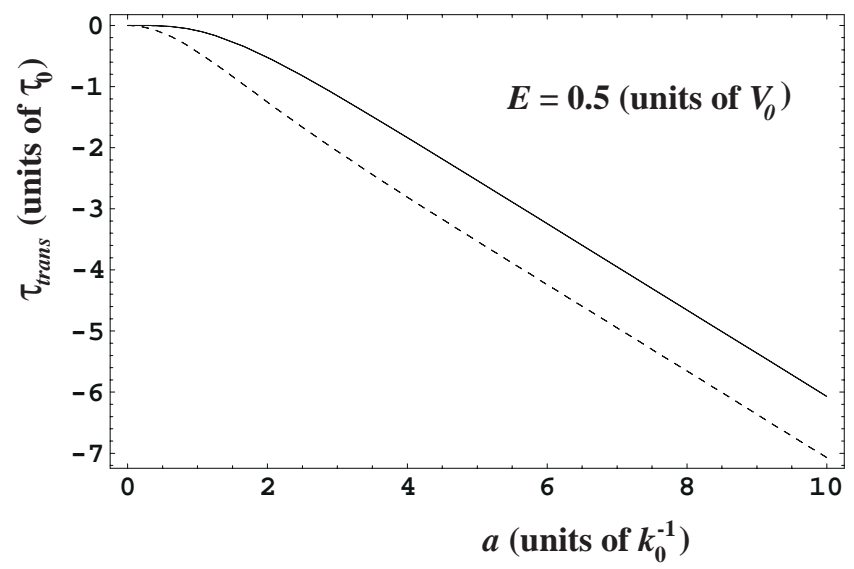

FIG. 4. Transmission time $\tau_{\text {trans }}$ (units of $\tau_{0}$ ) for the rectangular barrier as a function of barrier width $a$ (units of $k_{0}^{-1}$ ) for an energy of $E=0.5$ (units of $V_{0}$ ). The solid line is our result in Eq. (37) and the dashed line is the result of Pollak and Miller in Eq. (39). 
dauer [54]. The Pollak-Miller time is similar to the complex time in Eq. (13), obtained from the principle of constant local tempus, except that it uses the incoming wave function at the entrance instead of the total wave function. Therefore, it can be obtained by merely setting the reflection amplitude $A$ equal to zero in Eqs. (34) and (36).

The Pollak-Miller phase time is calculated from the phase difference $\Delta \phi_{0}$ in Eq. (34) when $A$ is set equal to zero. From Eq. (15), the Pollak-Miller phase time for the rectangular barrier is [32]

$$
\tau_{\text {phase }}^{\mathrm{PM}}=\frac{2 \kappa a k^{2}\left(\kappa^{2}-k^{2}\right)+\sinh (2 \kappa a)}{2 \kappa k\left[(2 \kappa k)^{2}+\sinh ^{2}(\kappa a)\right]} .
$$

The Pollak-Miller phase time $\tau_{\text {phase }}^{\mathrm{PM}}$ is plotted as the dashed curve in Fig. 1 as a function of the energy $E$, and in Fig. 2 as a function of barrier width $a$. Figure 2 shows the "Hartman effect" [52] in the saturation of the tunneling time for $a k_{0}$ $>5$, with a value $\tau_{\text {phase }}^{\mathrm{PM}}=(\kappa k)^{-1}$ from Eq. (38). Therefore, the ratio $\tau_{\text {phase }}^{\mathrm{PM}} / \tau_{\text {phase }}=2$ in Fig. 2 for $a k_{0}>5$ from Eq. (35). From Eq. (38), the initial slope of $\tau_{\text {phase }}^{\mathrm{PM}}$ in Fig. 2 is $\left[k^{2}\left(\kappa^{2}\right.\right.$ $\left.\left.-k^{2}\right)+1\right]\left(4 \kappa^{2} k^{3}\right)^{-1}$. The ratio of the initial slopes for $\tau_{\text {phase }}^{\mathrm{PM}}$ and $\tau_{\text {phase }}$ gives $\tau_{\text {phase }}^{\text {PM }} / \tau_{\text {phase }}=\left[k^{2}\left(\kappa^{2}-k^{2}\right)+1\right]\left(2 \kappa^{2} k^{2}\right)^{-1}$ for $a k_{0} \ll 1$. For the value of the energy $E=0.5$ used in Fig. 2 , the initial slope of $\tau_{\text {phase }}^{\mathrm{PM}}$ is $\sqrt{2}$ and the ratio $\tau_{\text {phase }}^{\mathrm{PM}} / \tau_{\text {phase }}$ $=2$ for $a k_{0} \ll 1$.

The Pollak-Miller transmission time is obtained from Eq. (36) by setting $A$ equal to zero. Therefore the Pollak-Miller time for the rectangular barrier is [32]

$$
\tau_{\text {trans }}^{\mathrm{PM}}=-\frac{\kappa a k^{2} \sinh (2 \kappa a)+2\left(\kappa^{2}-k^{2}\right) \sinh ^{2}(\kappa a)}{(2 \kappa k)^{2}\left[(2 \kappa k)^{2}+\sinh ^{2}(\kappa a)\right]} .
$$

The Pollak-Miller transmission time $\tau_{\text {phase }}^{\mathrm{PM}}$ is plotted as the dashed curve in Fig. 3, as a function of the energy $E$, and in Fig. 4 as a function of barrier width $a$. The negative values for $\tau_{\text {phase }}^{\mathrm{PM}}$ seem to be unphysical. Equation (39) shows that $\tau_{\text {phase }}^{\mathrm{PM}}$ is linear in $a$ with a slope of $-(2 \kappa)^{-1}$ for widths $a k_{0} \gg 1$. Therefore, $\tau_{\text {trans }}$ and $\tau_{\text {phase }}^{\mathrm{PM}}$ are parallel, as shown in Fig. 4.

\section{Larmor time}

The Larmor tunneling time [31] for a spin-1/2 particle propagating in the $y$ direction, with spin polarized in the $x$ direction, in an infinitesimal magnetic field in the $z$ direction, can be written in a complex form [32] for a rectangular barrier of height $V_{0}$ as

$$
\tau_{L}=-i \hbar \frac{\partial \ln D}{\partial V_{0}}
$$

The $y$ component of the Larmor transmission time is

$$
\tau_{y}=-\operatorname{Re} \tau_{L}=-\hbar \frac{\partial \Delta \phi_{0}}{\partial V_{0}}
$$

where it is necessary to use the negative of the real part, and the $z$ component is

$$
\tau_{z}=\operatorname{Im} \tau_{L}=-\frac{\hbar}{2} \frac{\partial \ln T}{\partial V_{0}} .
$$

Leavens and Aers [32] compare the Pollak-Miller [25] phase and transmission times with the corresponding Larmor times [31] for the rectangular barrier, so this is not done here.

\section{E. Numerical example}

A simple numerical application can be made using the heterostructure potential barrier parameters in GaAs $/ \mathrm{Al}_{0.3} \mathrm{Ga}_{0.7} \mathrm{As} / \mathrm{GaAs}$ [55]. The height is $V_{0} \simeq 0.3 \mathrm{eV}$ and a typical width is $a \simeq 50 \AA$. The effective mass ratio of the electron in GaAs is $m_{\mathrm{eff}} / m_{e} \simeq 0.067$. For these values, the dimensionless width of the potential is $a \simeq 3.63$ in units of $k_{0}^{-1}=13.77 \AA$ and the characteristic time is $\tau_{0}=\hbar / V_{0}$ $\simeq 2.19 \mathrm{fs}$.

\section{CONCLUSION}

We give a formulation of a traversal time in quantum mechanics using a principle of constant local tempus. The tempus operator is canonically conjugate to the energy operator [21], and has its roots in classical Hamiltonian theory $[19,20]$. Together the tempus operator and the energy operator satisfy the canonical commutation relation. In the representation in which the energy operator is $\hat{E}=E$, the tempus operator is $\hat{T}=i \hbar \partial / \partial E$, which acts only on the energy parameter $E$ in a stationary-state wave function. In the classical limit, the "principle of constant local tempus" gives the classical result obtained from Hamilton-Jacobi theory $[8,24]$. For a stationary-state wave function $\psi$ that depends on the energy, the local value of the tempus operator is $\hat{T} \psi / \psi$. The principle gives directly an "inverse quasitrajectory" $t(x)$, from which a traversal time $\tau=\Delta t=t\left(x_{2}\right)-t\left(x_{1}\right)$ is defined as the difference in time at $x_{2}$ and at $x_{1}$. For an energy beneath the top of a barrier, this method gives a complex tunneling time that has a real part equal to a phase time and an imaginary part equal to a transmission time.

Our method gives a result similar to the complex tunneling time obtained by Pollak and Miller [25] from a flux-flux correlation function. The Pollak-Miller time was also derived by Leavens and Aers [32] from the energy sensitivity of the transmission amplitude, a method suggested by Büttiker and Landauer [53] and further analyzed by Martin and Landauer [54]. Recently, an experiment on frustrated total internal reflection of light [26] used the complex Pollak-Miller time and showed how its real and imaginary parts could be interpreted [3]. The difference between our tunneling time and the Pollak-Miller tunneling time [25] is that, in our approach, the total wave function at the entrance $x_{1}$ of the barrier is used, whereas Pollak and Miller use only the incoming wave function. Only further experiments to measure tunneling times can show whether our tunneling time will be applicable $[29,30]$. 


\section{ACKNOWLEDGMENTS}

V. C. A.-N. H. I., and M. G. express their gratitude to the Department of Physics at the University of North Texas for hospitality during their visit. V. C. A.-N. acknowledges a grant from FAPESP, Brazil. D. H. K. would like to thank Professor R. Chiao and Professor J. Q. Liang for helpful discussions concerning tunneling times.
[1] R. Landauer and Th. Martin, Rev. Mod. Phys. 66, 217 (1994).

[2] E. H. Hauge and J. A. Støvneng, Rev. Mod. Phys. 61, 91 (1989).

[3] R. Chiao and A. M. Steinberg, in Progress in Optics, edited by E. Wolf (North-Holland, Amsterdam, 1997), Vol XXXVII, pp. 345-405.

[4] M. Jonson, in Quantum Transport in Semiconductors, edited by D. K. Ferry and C. Jacobini (Plenum, New York, 1985), pp. 193-238.

[5] A. P. Jauho, in Hot Carriers in Semiconductor Nanostructures: Physics and Applications, edited by J. Shah (Academic, Boston, 1992), pp. 121-151.

[6] P. Ghose, Testing Quantum Mechanics on New Ground (Cambridge University Press, Cambridge, England 1999), Chap. 10.

[7] J. G. Muga and C. R. Leavens, Phys. Rep. 338, 353 (2000).

[8] P. R. Holland, The Quantum Theory of Motion (Cambridge University, Cambridge, 1993), Chaps. 2, 3, and 5.5.

[9] C. R. Leavens and G. C. Aers, in Scanning Tunneling Microscopy III, edited by R. Wiesendanger and H.-J. Güntherodt (Spring-Verlag, Berlin, 1993), pp. 105-140.

[10] C. R. Leavens, Found. Phys. 23, 229 (1995).

[11] C. R. Leavens, Phys. Rev. A 58, 840 (1998).

[12] C. R. Leavens, in Tunneling and Its Implications, edited by D. Mugnai, A. Ranfagni, and L. S. Schulman (World Scientific, Singapore, 1997), pp. 100-120.

[13] W. R. McKinnon and C. R. Leavens, Phys. Rev. A 51, 2748 (1995).

[14] C. R. Leavens, Phys. Lett. A 197, 88 (1995).

[15] D. Sokolovski and L. M. Baskin, Phys. Rev. A 36, 4604 (1987)

[16] D. Sokolovski and J. N. L. Connor, Phys. Rev. A 42, 6512 (1990).

[17] D. Sokolovski and J. N. L. Connor, Phys. Rev. A 44, 1500 (1991).

[18] D. Sokolovski and J. N. L. Connor, Phys. Rev. A 47, 4677 (1993).

[19] D. H. Kobe, Am. J. Phys. 61, 1031 (1993).

[20] D. H. Kobe, Eur. J. Phys. 14, 262 (1993).

[21] D. H. Kobe and V. C. Aguilera-Navarro, Phys. Rev. A 50, 933 (1994).

[22] L. Cohen, Phys. Lett. A 212, 315 (1996).

[23] J. G. Muga, J. P. Palao, and R. Sala, Phys. Lett. A 238, 90 (1998).

[24] H. Goldstein, Classical Mechanics, 2nd ed. (Addison-Wesley, Reading, MA, 1980), Chaps. 9 and 10.

[25] E. Pollak and W. H. Miller, Phys. Rev. Lett. 53, 115 (1984). E. Pollak, J. Chem. Phys. 83, 1111 (1985).
[26] Ph. Balcou and L. Dutriaux, Phys. Rev. Lett. 78, 851 (1997).

[27] D. Sokolovski, S. Brouard, and J. N. L. Connor, Phys. Rev. A 50, 1240 (1994).

[28] S. Brouard, R. Sala, and J. G. Muga, Phys. Rev. A 49, 4312 (1994).

[29] R. Landauer, Nature (London) 341, 567 (1989).

[30] Tunneling and its Implications, edited by D. Mugnai, A. Ranfagni, and L. S. Schulman (World Scientific, Singapore, 1997).

[31] M. Büttiker and R. Landauer, Phys. Rev. Lett. 49, 1739 (1982). M. Büttiker, Phys. Rev. B 27, 6178 (1983).

[32] C. R. Leavens and G. C. Aers, Solid State Commun. 63, 1101 (1987).

[33] W. Pauli, General Principles of Quantum Mechanics (Springer-Verlag, Berlin, 1980), p. 63.

[34] E. A. Galapon, e-print quant-ph/9908033; and e-print quant-ph/0001062.

[35] I. L. Egusquiza and J. G. Muga, Phys. Rev. A 61, 012104 (2000).

[36] E. Fick and F. Engelmann, Z. Phys. 175, 271 (1963).

[37] F. Engelmann and E. Fick, Z. Phys. 178, 551 (1964).

[38] V. S. Olkhovsky and E. Recami, Phys. Rep. 214, 339 (1992).

[39] P. A. M. Dirac, The Principles of Quantum Mechanics, 4th ed. (Oxford University, London, 1958), pp. 84-89.

[40] J. Garcia-Bondía, Am. J. Phys. 58, 88 (1990).

[41] M. Razavy, Am. J. Phys. 35, 955 (1967).

[42] M. Razavy, Nuovo Cimento 63B, 271 (1969).

[43] M. Razavy, Can. J. Phys. 49, 3075 (1971).

[44] J. León, J. Julve, P. Pitanga, and F. J. de Urríes, Phys. Rev. A 61, 062101 (2000).

[45] V. S. Olkhovsky, E. Recami, and A. J. Gerasimchuk, Nuovo Cimento A 22, 263 (1974).

[46] V. S. Olkhovsky and E. Recami, Lett. Nuovo Cimento 4, 1165 (1970).

[47] E. Recami, in The Uncertainty Principle and Foundations of Quantum Mechanics, edited by W. C. Price and S. S. Chissick (Wiley, New York, 1977), pp. 21-28.

[48] E. Recami, W. A. Rodriguez, Jr., and P. Smrz, Hadronic J. 6, 1773 (1983).

[49] E. P. Wigner, Phys. Rev. 98, 145 (1955).

[50] F. T. Smith, Phys. Rev. 118, 349 (1960).

[51] D. Bohm, Quantum Theory (Prentice-Hall, New York, 1951), pp. 260-261, reprinted: (Dover, New York, 1989).

[52] T. E. Hartman, J. Appl. Phys. 33, 3427 (1962).

[53] M. Büttiker and R. Landauer, Phys. Scr. 32, 429 (1985).

[54] Th. Martin and R. Landauer, Phys. Rev. A 47, 2023 (1993).

[55] B. Lee, Superlattices Microstruct. 14, 295 (1993). 